\title{
Dose-Titrated Vasopressin V2 Receptor Antagonist Improves Renoprotection in a Mouse Model for Autosomal Dominant Polycystic Kidney Disease
}

\author{
Debbie Zittema $^{\mathrm{a}} \quad$ Irina B. Versteeg ${ }^{\mathrm{a}}$ Ron T. Gansevoort ${ }^{\mathrm{a}}$ Harry van Goor $^{\mathrm{b}}$ \\ Emile de Heer ${ }^{c}$ Kimberley A.M. Veraarc Dorien J.M. Peters ${ }^{d}$ Esther Meijer ${ }^{\mathrm{a}}$ \\ Departments of ${ }^{\mathrm{a}}$ Nephrology and ${ }^{\mathrm{b}}$ Pathology, University Medical Center Groningen, University of Groningen, Groningen, \\ and Departments of ${ }^{\mathrm{C}}$ Pathology and ${ }^{\mathrm{d}}$ Human Genetics, Leiden University Medical Center, Leiden, The Netherlands
}

\begin{abstract}
Key Words
Autosomal dominant polycystic kidney disease mouse model · Aquaresis - Renoprotective effect - Titrated dose regimen - Total kidney volume - Vasopressin $\mathrm{V} 2$ receptor antagonist
\end{abstract}

\begin{abstract}
Background: In autosomal dominant polycystic kidney disease, renoprotective treatment with a vasopressin V2 receptor antagonist (V2RA) is given in a fixed dose (FD). Disease progression and drug habituation could diminish treatment efficacy. We investigated whether the renoprotective effect of the V2RA can be improved by dose titration of the V2RA aiming to maintain aquaresis at a high level. Methods: The V2RA OPC-31260 was administered to Pkd1-deletion mice in an FD $(0.1 \%)$ or in a titrated dose (TD, up to $0.8 \%$ when drinking volume dropped). Total kidney weight (TKW) and cyst ratio were investigated and compared to non-treated $P k d 1$ deletion mice. Treatment was started early or late (21 or 42 days postnatal). Results: Water intake was significantly higher throughout the experiment in the TD compared to the FD group. FD treatment that was initiated early reduced TKW and cyst ratio but lost its renoprotective effect later during the experiment. In contrast, TD treatment was able to maintain the renoprotective effect. TD treatment, however, was also associated with a higher early termination rate in com-
\end{abstract}

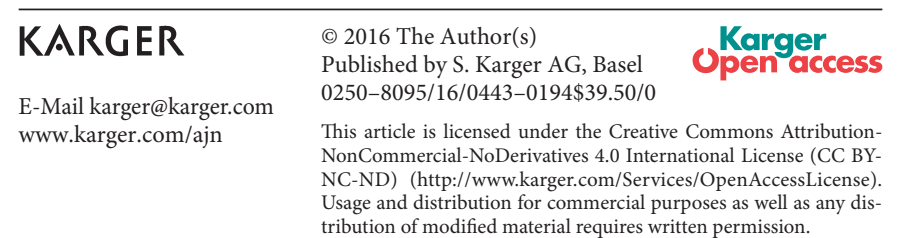

parison with FD treatment. Late start of treatment (FD or TD) did not show a renoprotective effect. Conclusions: Titration of a V2RA aimed to maintain aquaresis at a high level showed a better renoprotective effect compared to FD administration. However, this treatment regimen was poorly tolerated and did not overcome treatment unresponsiveness when started later in the disease.

(c) 2016 The Author(s)

Published by S. Karger AG, Basel

\section{Introduction}

Autosomal dominant polycystic kidney disease (ADPKD) is a hereditary kidney disease with an estimated point prevalence of clinically diagnosed cases of 3 per 10,000 subjects in the general population [1]. ADPKD is characterized by cyst formation in both kidneys and renal function loss, leading to end-stage kidney failure in most affected patients.

The pathogenesis of ADPKD is complex and not fully understood. However, it is generally acknowledged that elevated intracellular levels of adenosine $3^{\prime}: 5^{\prime}$-cyclic monophosphate (cAMP), formed by adenylyl cyclase, are part of the pathway leading to renal tubular cell proliferation and increased chloride-driven fluid secretion by these cells. Both processes contribute to cyst formation and growth [2]. Studies using in vitro ADPKD cells dem- 
Fig. 1. Experimental design.

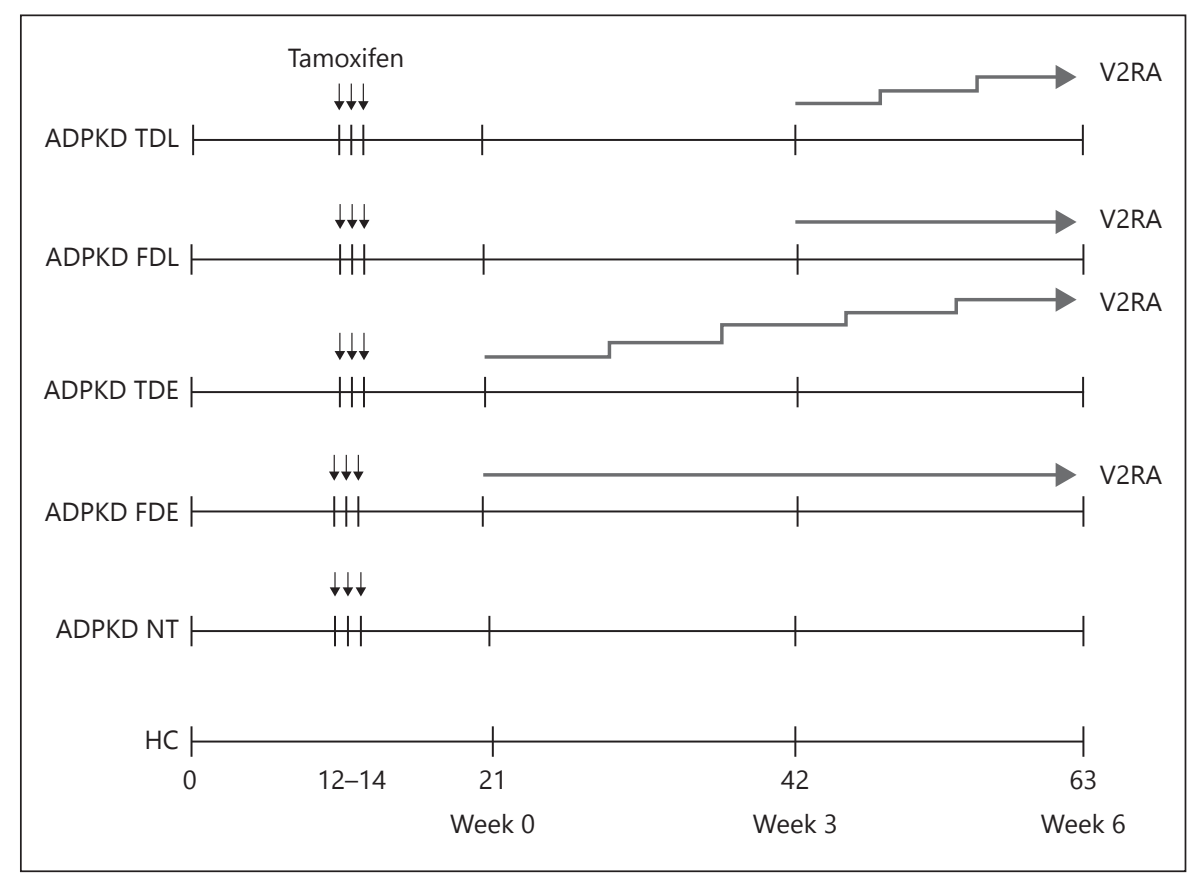

onstrated that administration of cAMP, or an adenyl cyclase agonist like vasopressin, leads to cell proliferation [3] and fluid accumulation [4]. Moreover, blocking the vasopressin V2 receptor in animal models attenuated disease progression [5-10]. In line with these reports, a recent multicenter randomized controlled trial showed that a vasopressin V2 receptor antagonist (V2RA) slowed the increase in total kidney volume and the decline in kidney function over a 3-year period in patients with relatively early stage ADPKD [11]. The most frequently reported adverse events were polyuria and polydipsia. These adverse events can be expected, as water reabsorption in the collecting duct, enabled by aquaporine- 2 water channels, relies on the stimulation of the V2 receptor [12]. The beneficial renoprotective effect of V2RA treatment is therefore accompanied by a strong aquaretic response. In addition, potential hepatotoxicity was observed in individual cases.

Optimum dosage of the drug is yet debatable. As of now, all studies investigated a fixed-dose (FD) treatment regimen. However, it has been suggested that disease severity and treatment duration may interfere with treatment efficacy, as indicated by a decline in aquaretic response to V2RAs in later stage disease and during prolonged treatment $[8,13,14]$. We hypothesized therefore that the renoprotective effect of the V2RA can be optimized when the dose of the V2RA is increased during treatment, aiming to maintain aquaresis at a high level, to overcome drug habituation. We studied this hypothesis in a $P k d 1$-deletion mouse model with high face validity to the human situation, in early- and later-stage disease.

\section{Material and Methods}

\section{Experimental Animals}

For this experiment, the tamoxifen-inducible kidney epithelium-specific $P k d 1$-deletion mouse was used. Upon administration of tamoxifen, a genomic fragment containing exons 2-11 of the $P k d 1$ gene is specifically deleted in renal epithelial cells and cysts are formed. This inducible Pkd1-deletion mouse model (tamKspCad-CreER $\left.{ }^{\mathrm{T} 2} ; \mathrm{Pkd}^{\mathrm{lox} 2-11 / \mathrm{lox} 2-11}\right)$ has been described earlier [8, $15]$. We administered tamoxifen per gavage $(0.22 \mathrm{mg}$ dissolved in ethanol in $0.30 \mu \mathrm{l}$ sunflower oil; Sigma-Aldrich, St. Louis, Mo., USA) for 3 consecutive days starting at postnatal day 12. Gene deletion was induced early in life to induce cyst formation in collecting ducts and distal tubules and to develop a more rapid progressive form of ADPKD. All mice were genotyped using a polymerase chain reaction method and had a confirmed homozygote $P k d 1$ deletion. Male and female animals were stratified over the study groups and analyzed together as no difference in rate of disease progression has been noted in this model across sex.

\section{Experimental Design}

Figure 1 displays an overview of the experimental design. At 3 weeks of age, tamoxifen treated mice were divided into a notreatment (NT) group and 4 treatment groups: FD early start of treatment (FDE), titrated dose (TD) early start of treatment (TDE), FD late start of treatment (FDL), and TD late start of treatment (TDL; table 1). Mice not treated with tamoxifen served as a healthy 
Table 1. Number of $P k d 1$-deletion and healthy control mice randomized across the various experimental groups and at the time points of sacrifice. Body weight and gender were not different between the experimental groups at randomization. However, the early termination rate was higher in the titrated dose early start of treatment group

\begin{tabular}{|c|c|c|c|c|c|c|c|}
\hline \multirow[t]{2}{*}{ Groups } & \multirow[t]{2}{*}{ Total, $\mathrm{n}$} & \multirow[t]{2}{*}{ Week $0, \mathrm{n}$} & \multicolumn{2}{|c|}{ Week 3} & \multicolumn{2}{|c|}{ Week 6} & \multirow[t]{2}{*}{ ET: total, n (\%) } \\
\hline & & & $\mathrm{n}$ & ET & $\mathrm{n}$ & ET & \\
\hline $\mathrm{HC}$ & 17 & 6 & 4 & 0 & 7 & 0 & $0 / 17(0)$ \\
\hline NT & 37 & 10 & 11 & 0 & 16 & 2 & $2 / 37(5)$ \\
\hline FDE & 21 & & 11 & 0 & 10 & 0 & $0 / 21(0)$ \\
\hline TDE & 25 & & 9 & 0 & 16 & 6 & $6 / 25(24)$ \\
\hline FDL & 12 & & & & 12 & 1 & $1 / 12(8)$ \\
\hline TDL & 11 & & & & 11 & 1 & $1 / 11(9)$ \\
\hline Total & 123 & 16 & 35 & 0 & 72 & 10 & $10 / 123(8)$ \\
\hline
\end{tabular}

control group (HC). This time point serves as baseline, that is, as week 0 of the experiment.

The V2RA OPC-31260 (Mozavaptan) was added to ground rodent chow (AB Diets BV, RMH-B starch, 2103) at a starting dose of $0.1 \%$ and was given to all mice on treatment. This $0.1 \%$ dose is equivalent to the dose used in literature $[6,9]$ and has been used in this particular $P k d 1$ mouse model by our research group before [8].

According to welfare regulations, it was not possible to house mice individually at the start of the study because of their small size at 3 weeks of age. Therefore, mice were housed with an average of 3 mice per cage. Animals, water and food where weighed 2 times a week. Water intake of the cage was divided by the number of mice per cage, the number of days between 2 consecutive measurements and weight in order to obtain an average water intake per animal per day. Water intake was adjusted for body weight because mice grew to maturity during the experiment and this is accompanied by an increase in water intake.

When water intake of mice in the TD treatment groups decreased with more than $10 \%$ compared to the average water intake during the first 2 consecutive measurements in the first week, the dose of the V2RA was increased to $0.2 \%$. When again drinking volume dropped with $10 \%$ compared to the first 2 consecutive measurements in the first week, the dose was again increased. The dose scheme of the V2RA was: $0.1,0.2,0.4,0.6$, and $0.8 \%$, respectively (fig. 1). Mice in the FD treatment groups continued treatment with $0.1 \%$ V2RA. Treatment was started either at 21 days postnatal (day 0 of the experiment, 'early') or at 42 days postnatal (week 3 of the experiment, 'late'). At day 0, weeks 3 and 6 of the experiment mice were sacrificed. Early termination (ET) criteria were weight loss of $>20 \%$ or change in skin, hair or locomotion. When mice were sacrificed $>1$ week before the planned sacrifice, they were counted as ET and excluded from all analyses (table 1).

All experiments were approved by the local animal experiment committee of the University Medical Center Groningen and by the Committee Biotechnology in Animals of the Dutch Ministry of Agriculture.

\section{Data Collection}

Twenty-four-hour urine output was obtained for individual mice by placing them in metabolic cages before mice were sacrificed at week 3 or 6 of the experiment. Subsequently, the animals were weighed and anesthetized with isoflurane gas $(0.5 \%$ isoflu- rane with a flow of $0.6 \mathrm{l} / \mathrm{min}$ ). Blood was obtained by cardiac puncture for determination of plasma electrolytes, creatinine, urea, aspartate transaminase (AST), alanine transaminase (ALT) and bilirubin. Both kidneys were removed and kidney weight was measured on a precision scale. Half of the right kidney was placed into formaldehyde and tissues were embedded in paraffin for histomorphometry and immunohistochemistry.

\section{Plasma and Urine Analysis}

Sodium, potassium, urea and creatinine were measured in plasma and urine, using Kodak Ektachem dry chemistry (Modular P, Roche, Mannheim, Germany). Urine osmolality was calculated as $2 \times$ (urine sodium concentration + urine potassium concentration) + urine urea concentration [16]. Plasma osmolality was calculated using the formula: $1.9 *$ (plasma sodium concentration + plasma potassium concentration) + plasma glucose concentration + plasma urea concentration * $0.5+5$ [17].

\section{Immunohistochemistry and Histomorphometric Analysis}

Transverse tissue sections $(4 \mu \mathrm{m})$, including cortex, medulla and papilla, were stained with periodic acid Schiff and hematoxylin-eosin to measure cyst ratio [8] and with sirius red collagen stain to measure the extent of fibrosis [18]. The total cyst and fibrosis areas were quantified using Aperio Image Scope software version 9.1.772.1570 (Aperio Technologies Inc., Vista, Calif., USA). Kidneys were outlined manually, pyelum not included. Cyst ratio was calculated by dividing total cysts area by total tissue area and multiplied by $100 \%$ to obtain a cyst ratio, expressed as percentage. The total intensity of fibrosis staining was divided by the total intensity on the slide (both positive and negative) and multiplied by $100 \%$ to obtain a percentage of fibrosis. The investigator performing these measurements was blinded to disease and treatment status. To calculate the medulla/cortex cyst ratio, 5 squares in both medulla and cortex were drawn and the average cyst ratio was determined for both areas separately to calculate the medulla/cortex ratio.

\section{Statistical Analyses}

Differences between 2 groups were tested using a non-parametric independent sample test (Mann-Whitney) and differences between all groups were tested using a Kruskal-Wallis test or Pearson's chi-square test. Analyses were performed using the statistical 


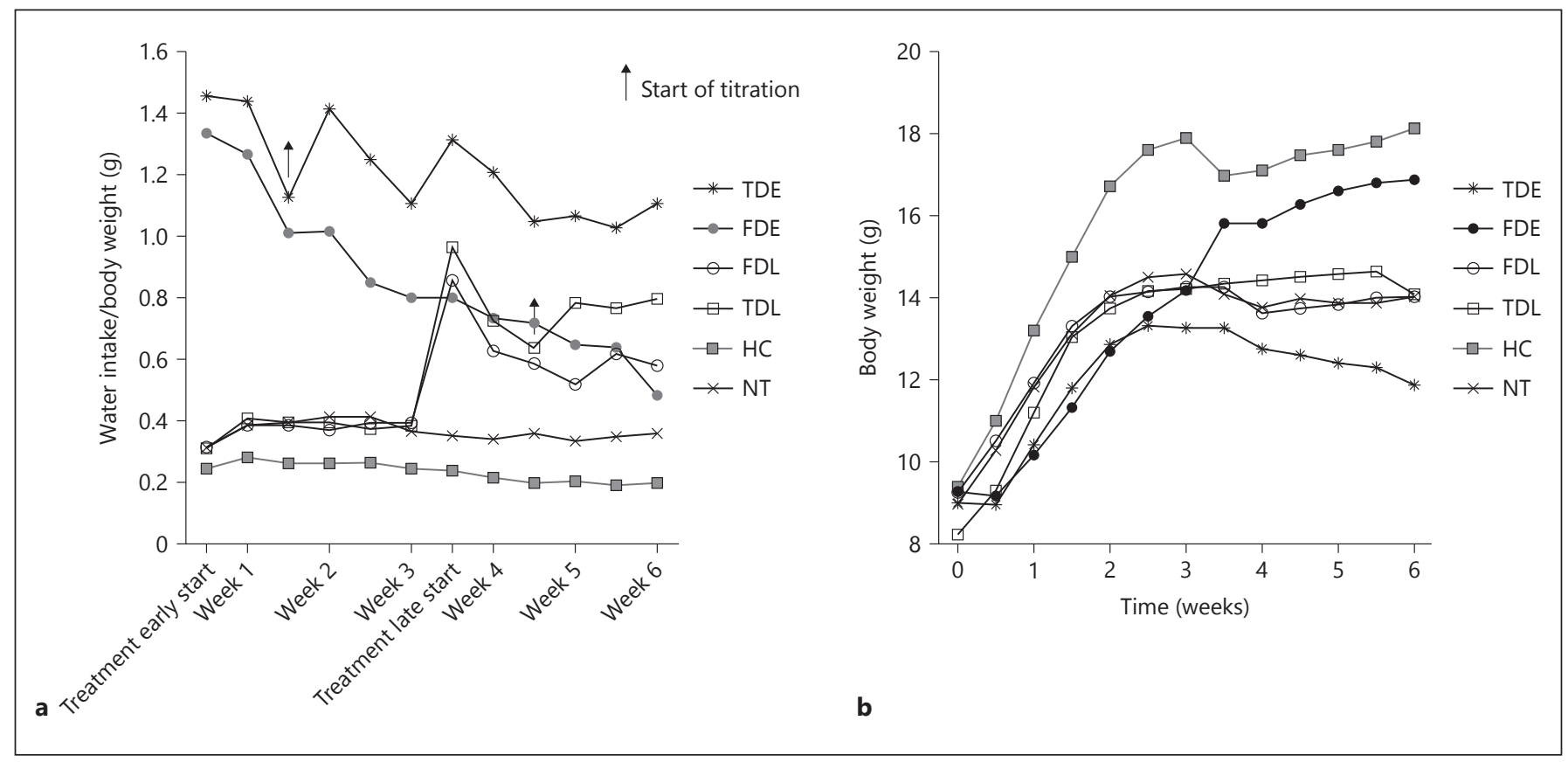

Fig. 2. Mean water intake (a) and body weight (b) of mice in the various treatment groups during the experiment. The arrow indicates the time-point at which dose titration of the V2RA was started.

package IBM SPSS statistics version 20.0 (International Business Machines Corp., Chicago, Ill., USA). A 2-sided $\mathrm{p}<0.05$ was considered to indicate statistical significance.

\section{Results}

At the start of the experiment, $P k d 1$-deletion mice were randomized over the following experimental groups: NT, FDE, FDL, TDE, and TDL. Table 1 shows the number of mice randomized to the various treatment groups and mice that needed to be sacrificed because they met the ET criterion of weight loss $>20 \%$. Figure 1 gives an overview of the experimental design. Body weight and gender were not different between the experimental groups at randomization ( $p=0.39$ and $p=0.79$, respectively). Moreover, at start of the study body weight and total kidney weight (TKW), of mice that were sacrificed at week 0 , were similar between $\mathrm{HC}$ male and female mice ( $\mathrm{p}=0.17$ and $\mathrm{p}=0.63$, respectively) and $P k d 1$-deletion male and female mice ( $\mathrm{p}=0.51$ and $\mathrm{p}=0.53$, respectively).

\section{Non-Treated ADPKD}

$P k d 1$-deletion mice developed cysts and kidney function deterioration during the experiment. Study parameters indicative for renal damage (TKW, cyst ratio, fibro- sis ratio, plasma creatinine and plasma urea) were all significantly increased in $P k d 1$-deletion animals compared to HCs at weeks 3 and 6 of the experiment (fig. 4; online suppl. tables 1 and 2; for all online suppl. material, see www.karger.com/doi/10.1159/000448693).

Water intake was significantly higher in non-treated Pkd1-deletion mice compared to HCs throughout the whole experiment, starting already at day $0(\mathrm{p}<0.001$; fig. 2). Together with a significant lower 24-hour urine osmolality and an increased plasma osmolality at weeks 3 and 6 of the experiment in these non-treated $P k d 1$-deletion mice group (fig. 3), this suggests an early urine concentrating defect. Although bodyweight was similar at randomization, $P k d 1$-deletion mice showed lower growth rates compared to $\mathrm{HC}$ mice with a significantly lower bodyweight at week $3(\mathrm{p}=0.001)$ and $6(\mathrm{p}=0.01)$, while food intake (adjusted for body weight) was similar both at 3 weeks $(p=1.00)$ and 6 weeks $(p=0.84)$ of the experiment.

\section{FDE Treatment}

Water intake increased after the initiation of V2RA treatment. However, the aquaretic response in the FD treatment group waned during the course of treatment. At week 3, water intake was still higher in treated mice compared to non-treated mice $(\mathrm{p}<0.001)$, but at 


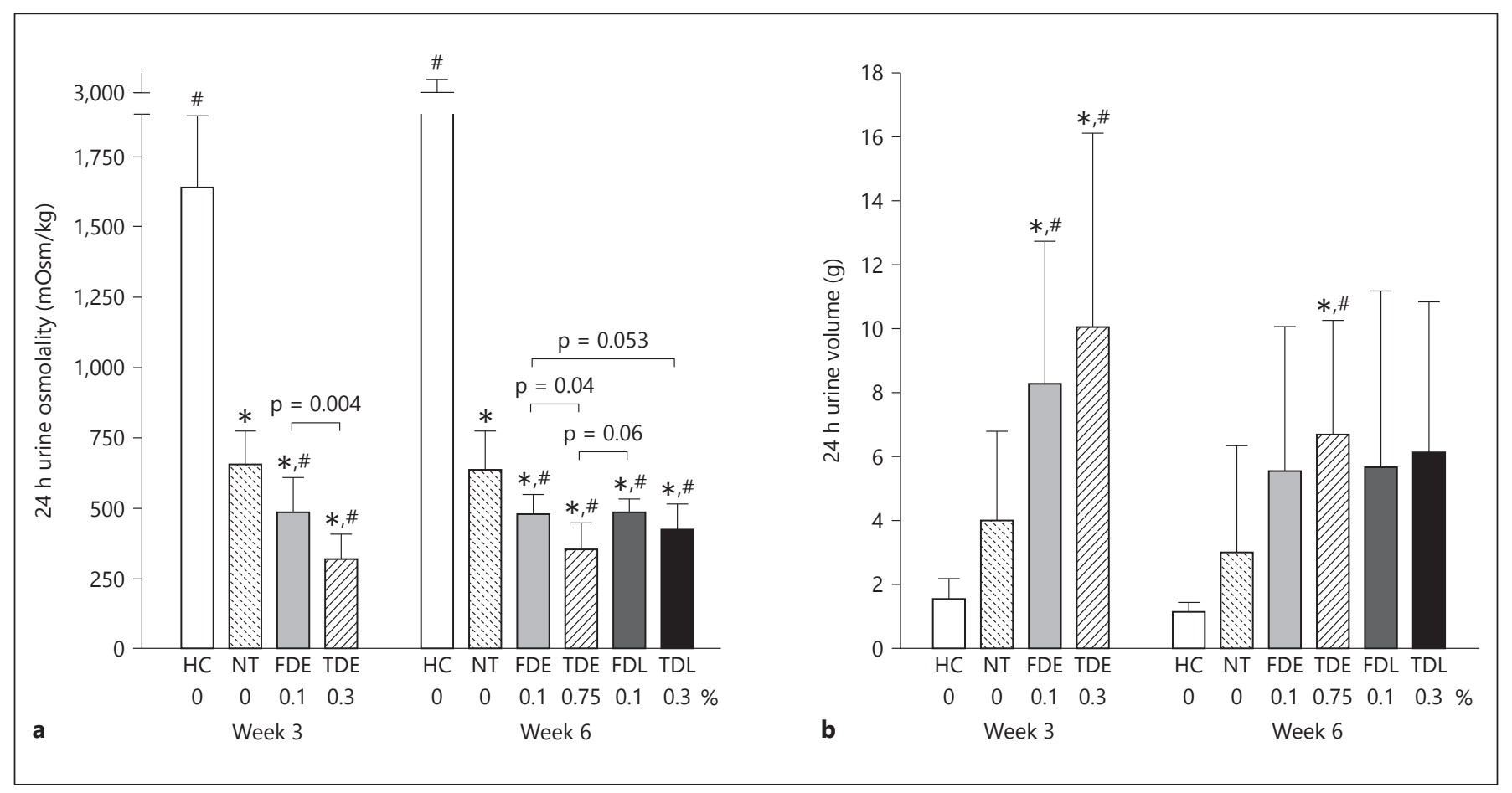

Fig. 3. Twenty-four-hour urine osmolality (a) and 24-hour urine volume (b) at weeks 3 and 6 of the experiment. X-axis displays, besides the various treatment groups, the mean dose of the V2RA administration during 24-hour urine collection. Data are displayed as mean and SD. ${ }^{*} \mathrm{p}<0.05$ compared to the HC mice; ${ }^{*} \mathrm{p}<$ 0.05 compared to the non-treated $P k d 1$-deletion mice. week 6 this difference did not reach significance anymore $(\mathrm{p}=0.09$; fig. 2$)$. In line, the increase in 24-hour urine volume during treatment lost statistical significance (fig. 3). The osmolality of 24-hour urine, however, remained significantly lower at weeks $3(p=0.005)$ and $6(\mathrm{p}=0.004)$ of the experiment (fig. 3). Body weight was higher in the FD treatment group during the last weeks of the experiment compared to non-treated mice (fig. 2).

TKW was significantly lower in the FD treatment group compared to non-treated $P k d 1$-deletion mice at week 3 of the experiment $(\mathrm{p}=0.02)$. However, at week 6 , this difference also lost statistical significance $(\mathrm{p}=0.26$; fig. 4). Body weight in the FD treatment group compared to non-treated $P k d 1$-deletion mice was similar at week 3 $(p=0.16)$ and borderline significantly higher at week 6 of the experiment $(\mathrm{p}=0.05)$. Food intake (adjusted for body weight) was equal between these groups at week 3 ( $p=$ $0.56)$ but lower in the FD treatment group compared to non-treated $P k d 1$-deletion mice $(\mathrm{p}=0.04)$ at week 6 of the experiment. Cyst ratio, fibrosis ratio and plasma creatinine did not differ between the 2 groups at weeks 3 and 6 (online suppl. tables 1 and 2).

\section{TDE Treatment}

At start of the experiment (day 0), the dose of the V2RA in the titrated and FD treatment groups was the same and both groups showed a similar increase in water intake. After 1 week, water intake decreased by more than $>10 \%$ in both treatment groups. Per protocol, dose titration of the V2RA was started in the TDE treatment group, resulting in a higher V2RA dose (average mean dose of $0.75 \%$ V2RA at week 6 of the experiment, average overall dose of $0.38 \%, p<0.001$ ) and significantly higher water intake $(\mathrm{p}=0.04)$ and lower 24-hour urine osmolality (week 3: $\mathrm{p}=0.004$, week 6: $\mathrm{p}=0.04$ ) compared to the FD treatment group that still received $0.1 \%$ V2RA (fig. 2, 3). Body weight was lower in the TD treatment group compared to the FD treatment group $(\mathrm{p}=0.002)$ and nontreated $P k d 1$-deletion animals $(\mathrm{p}=0.04)$ at week 6 of the experiment (fig. 2). Food intake (adjusted for body weight) at this time point was similar in comparison to the FD treatment group $(\mathrm{p}=0.81)$ but lower in comparison to non-treated $P k d 1$-deletion mice ( $\mathrm{p}=0.01)$.

In contrast to mice in the FD treatment group, the renoprotective effect of the V2RA was seen throughout the experiment in the TD treatment group with a lower 


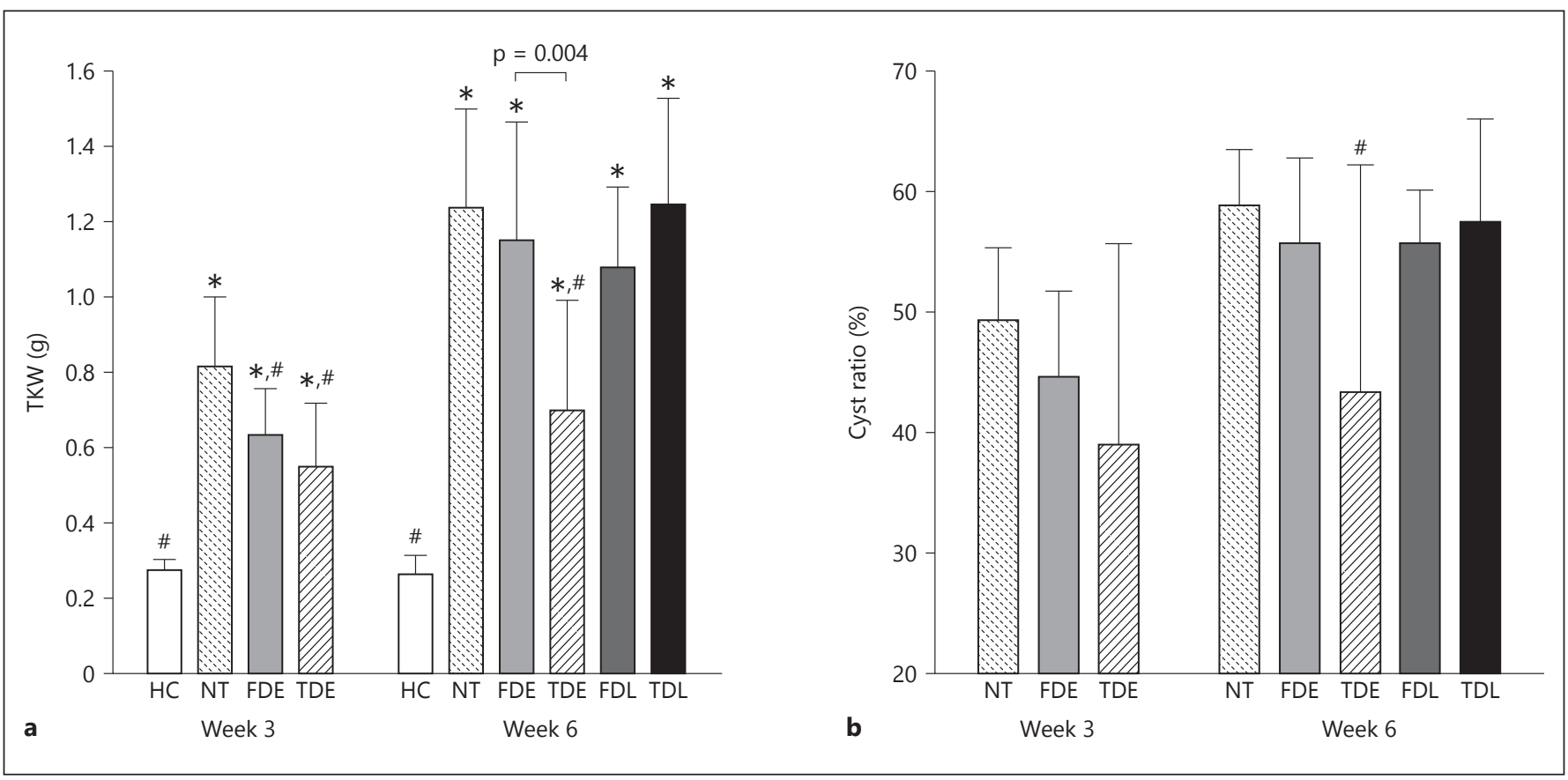

Fig. 4. TKW (a) and cyst ratio (b) at weeks 3 and 6 of the experiment. Data are displayed as mean and SD. ${ }^{*} \mathrm{p}<$ 0.05 compared to the HC mice; ${ }^{\#} \mathrm{p}<0.05$ compared to the non-treated $P k d 1$-deletion mice.

TKW at week $3(\mathrm{p}=0.004)$ and week $6(\mathrm{p}<0.001)$ and a lower cyst ratio at week 6 in comparison to non-treated $P k d 1$-deletion mice ( $\mathrm{p}=0.04$; fig. 4 ). When comparing the fixed and TD treatment groups, TKW was similar at week 3. However, at week 6 of the experiment TKW was significantly lower in the TD treatment group compared to the FD treatment group ( $\mathrm{p}=0.004$; fig. 4$)$. A similar trend was observed for cyst ratio, although the difference did not reach formal statistical significance $(p=0.17)$. Fibrosis ratio, plasma creatinine and plasma osmolality were similar (online suppl. tables 1 and 2).

When investigating the medulla/cortex cyst ratio in the non-treated $P k d 1$-deletion mice, in our model, cysts were first mainly located in the medulla. When disease progressed, the medulla/cortex ratio decreased significantly, indicating the growth of cortical cysts. At week 6 of the experiment, the medulla/cortex cyst ratio was higher in the TD treated group compared to that in the nontreatment group $(p=0.06)$. This was not the case for the FD treatment group. Furthermore, in the FD treatment group, the medulla/cortex cyst ratio declined significantly when comparing weeks 3 and $6(\mathrm{p}<0.001)$, while in the TD treatment group, the medulla/cortex cyst ratio remained more stable (fig. 5).

The TD treatment regimen was poorly tolerated during the second half of the experiment. When the V2RA dose was increased, the weight of mice decreased before stabilizing after a few days, resulting in a high number of ETs in the second half of the experiment ( 6 out of 16 mice in the TD treatment group versus 0 out of 10 mice in the FD treatment group; table 1). The mean TKW of early terminated mice in the TD treatment group was $0.56 \mathrm{~g}$. In comparison, the mean TKW of mice in the titrated treatment group and non-treated $P k d 1$-deletion mice at week 6 of the experiment was 0.70 and $1.24 \mathrm{~g}$, respectively.

As potential hepatotoxicity was observed in human studies involving V2RA treatment, several parameters reflecting liver function were investigated. The TD treatment regimen did not cause an elevation in AST, ALT or bilirubin compared with non-treated mice (178 vs. 238 $\mathrm{U} / \mathrm{l}, \mathrm{p}=0.92 ; 83$ vs. $83 \mathrm{U} / \mathrm{l}, \mathrm{p}=0.44 ; 6$ vs. $5 \mu \mathrm{mol} / \mathrm{l}, \mathrm{p}=$ 0.92 , respectively) or FD-treated mice (178 vs. $217 \mathrm{U} / \mathrm{l}$, $\mathrm{p}=0.89 ; 83$ vs. $57 \mathrm{U} / \mathrm{l}, \mathrm{p}=0.27 ; 6$ vs. $5 \mu \mathrm{mol} / \mathrm{l}, \mathrm{p}=0.40$, respectively). Furthermore, liver histology of mice treated with the TD of the V2RA was similar in comparison with non-treated and FD-treated mice.

\section{Early versus Late Start of Treatment}

When treatment with $0.1 \%$ V2RA treatment started late (FDL), that is, at week 3 of the experiment, water intake increased compared to non-treated $P k d 1$-deletion animals but was significantly lower compared to water 


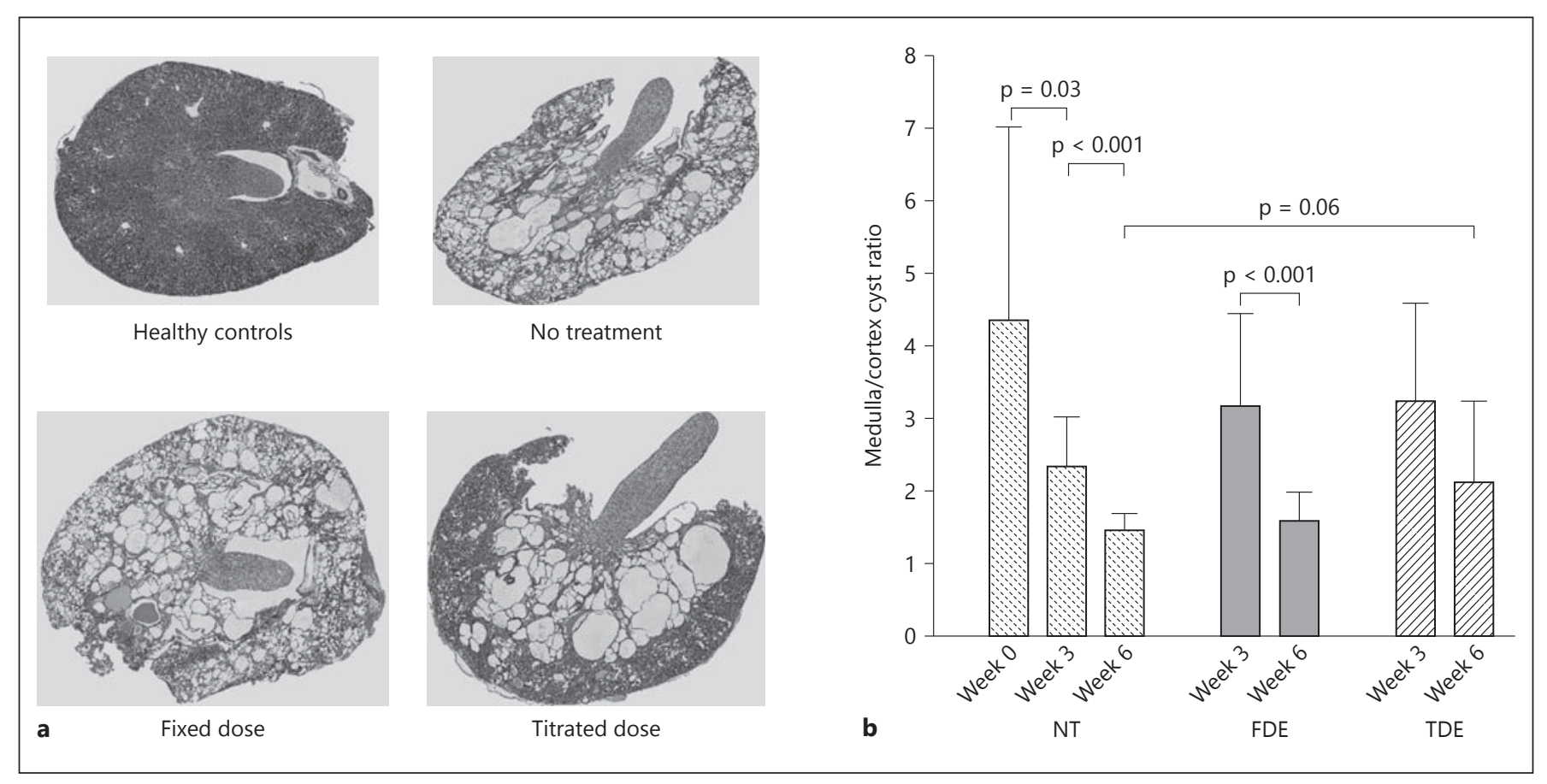

Fig. 5. Tissue morphology at week 6 of the experiment (a) and the medulla/cortex cyst ratio at weeks 0, 3 and 6 of the experiment (b). Data are displayed as mean and SD.

intake at the start of treatment in the early treatment groups $(\mathrm{p}=0.004)$. The TDL underwent a similar up-titration schedule compared with the TDE group during their first 3 weeks of treatment. Nevertheless, the average water intake in this group was significantly lower $(\mathrm{p}=$ 0.01 ) than that of mice in the early start of titration treatment group in their first weeks of the experiment.

Starting treatment later in the disease course, using a fixed or TD treatment regimen, did not have an effect on the various parameters for renal damage (TKW, cyst ratio, fibrosis ratio, and plasma creatinine and osmolality) in comparison with non-treated mice (online suppl. table 2 and fig. 4).

\section{Discussion}

In this experiment, we studied the effects of titration of a V2RA in an ADPKD mouse model. By aiming to maintain water intake at a high level, we tried to overcome potential habituation to the V2RA and improve the renoprotective efficacy of this drug in the early and later stage of the disease. During the experiment, the aquaretic response to dose titration was indisputable. Water intake was significantly higher throughout the experiment in mice that received the TD-treatment regimen compared to the FD-treatment regimen. This was associated with a renoprotective effect, also later in the disease. In our model, cyst formation starts predominantly in the distal tubular segments, whereas later on also, cysts arise in the cortex. In comparison to FD treatment, TD treatment had an inhibitory effect on cyst formation in the cortex as well, indicating the ongoing benefit of titration of the V2RA. Unfortunately, the renoprotective effect of V2RA titration was obtained at the cost of a higher ET rate in the titrated treatment group. Moreover, the titrated treatment regimen could not overcome the unresponsiveness of the V2RA when started in a later stage of the disease.

Previously, we hypothesized that when ADPKD progresses more, vasopressin is released from the pituitary gland, as a response to the impaired urine concentrating capacity that is observed in more severe form of the disease [19]. Indeed, we have found that copeptin, a surrogate marker for vasopressin, is positively associated with disease severity [20,21], which fits this hypothesis. Furthermore, during V2RA treatment, vasopressin levels increase $[8,22]$ and it has been suggested that the expression of the vasopressin $\mathrm{V} 2$ receptor is downregulated via the stimulation of the vasopressin V1 receptor [23, 24]. Taken together, this suggests that in more severe ADPKD, 
especially when treated with a V2RA, more AVP per receptor will be present and thus that a higher dose of the V2RA may be needed to result in effective receptor blockade. Indeed, our experiment shows that dose titration of the V2RA during disease progression results in a stronger aquaretic response and improved renoprotection when compared to an FD-treatment regimen. However, no effect of V2RA treatment was seen when initiated later in the disease. Dose titration (from 0.1 to $0.4 \%$ ) was not able to overcome this decreased efficacy for the cystic phenotype. A possible explanation is that during disease progression other cystogenic mechanisms not involving cAMP become more important, for instance, pathways involving growth factors or macrophages [2]. Loss of functioning nephrons may also explain the lack of efficacy when treatment is initiated later in the disease. Involvement of the vasopressin V2 receptor itself in treatment unresponsiveness could explain the less profound aquaretic response as well. Mechanisms that can be taken into consideration are for instance loss of vasopressin V2 receptors when cysts disconnect from the tubular system or vasopressin V2 receptor dedifferentiation. In line with this theory are results from our previous animal experiment that showed a decrease in V2-receptor mRNA expression over time in non-treated ADPKD animals [8].

The reason for the high dropout rate seen in mice on the high V2RA dose is not entirely clear from the present experiment. In contrast to what is found in humans [11], we did not find elevated liver enzymes indicative for hepatotoxicity of the V2RA in our mice. Histology of the liver was not different between the non-treated and treated $P k d 1$-deletion mice either. This is in line with experiments in mice reporting no toxicity after administration of a single $1,000 \mathrm{mg} / \mathrm{kg}$ dose, which is equivalent to our $0.8 \%$ [25]. In the present experiment we did, however, observe a decrease in body weight, especially in the mice that received the titrated treatment regimen. Kidney weight of these early terminated animals was significantly lower compared to the non-treated controls, indicating that disease progression per se is not a likely cause of this weight loss. We hypothesize that this weight loss is caused by their effort to drink up to 1.5 times their bodyweight per day, which may have caused inadequate food intake. Almost a quarter of the animals had to be sacrificed because of pre-specified ET criterion regarding weight loss. It is not likely that this is related to the fact that mice had polycystic kidney disease because in healthy rats and dogs, similar mortality rates have been described on a daily $1,000 \mathrm{mg} / \mathrm{kg}$ dose, likewise without a clear cause of death [25].

Titrated Vasopressin Blockade in ADPKD
Translating experimental data into clinical practice is difficult and should be done with caution. Our experiment shows, however, that a better therapeutic response can be achieved when higher doses of the V2RA are used. This suggests that dose titration of a V2RA in clinical practice, when disease progresses and the aquaretic response to the V2RA decreases, may be beneficial. However, this renoprotective effect was counteracted by significant side effects. Moreover, this rigorous treatment regimen did not overcome therapeutic unresponsiveness when started in a later stage of the disease. Animal experiments performed by other research groups did not describe a decrease in responsiveness during long-term treatment as we observed in our model $[6,7,9,10]$. In addition, results of a post-hoc analysis of the TEMPO 3:4 trial showed that a V2RA was similarly effective in reducing the rate of total kidney volume increase and eGFR decline in ADPKD patients with CKD stages 1 to 3a [26]. Many factors, including the experimental model, dosage of the V2RA and disease severity, may explain the differences between these studies. Regardless of the variance of the present results and previous data, clinical studies are needed to verify the efficacy and safety of long-term V2RA treatment, especially in ADPKD patients with a more advanced form of the disease (CKD stages $3 \mathrm{~b}$ and 4 ).

To enhance therapeutic efficacy, combination therapy could be of more use. Theoretically, somatostatin analogues induce a similar effect on intracellular cAMP production as V2RAs because both drug classes inhibit adenylyl cyclase, the enzyme that converts adenosine triphosphate into cAMP [27]. Interestingly, Hopp et al. [28] recently showed in a $P k d 1$ experimental model that combination treatment with a somatostatin analogue and a V2RA indeed led to lower cAMP levels and less cyst progression when compared to monotherapy with either of the drugs. A combination of these drugs may allow the use of lower doses of each single drug, thereby limiting adverse events, while optimizing renoprotective efficacy. These results are promising and seem to be a more logical direction for future research than applying higher doses monotherapy.

We acknowledge that this experiment has limitations. First, the rapidly progressive model used in this experiment does not exactly resemble the clinical situation where ADPKD is often a more slowly progressive disease. However, this model allowed us to investigate the effect of titration as well as start of treatment in the later stages of the disease. Second, it was not possible to house mice individually at the start of the study because of their small size at 3 weeks of age. We preferred early 
start of treatment over individually housed animals. Therefore, mice were housed with an average of 3 mice per cage and titration of the V2RA treatment was based on the average water intake of 3 mice. Third, bodyweight of the mice in the TD treatment group was lower compared to the other study groups. Because water intake remained higher in the TD treatment group compared to the other groups and responded to titration steps, a lower food intake and consequently, possibly a lower dose of the V2RA is not a concern. Nevertheless, recent studies in mice revealed that a lower food intake can also be protective in ADPKD [29,30]. Although food intake (adjusted for body weight) in the TD treatment group was similar after 6 weeks of study in comparison to the FD treatment group, body weight was markedly different between these study groups at the end of our experiment. We therefore cannot exclude a possible influence of food intake on disease progression. The strengths of this experiment include the unique experimental design that addresses a study question that has not been investigated previously, the use of a $P k d 1$-deletion model with high face validity to the human situation where ADPKD is caused by mutations in the PKD1 gene in
$85 \%$ of cases, and the potential clinical relevance of our findings.

In conclusion, titration of a V2RA aimed to maintain aquaresis at a high level, showed a better renoprotective effect compared to FD administration in this ADPKD mouse model. However, this TD treatment regimen was poorly tolerated and did not overcome treatment unresponsiveness when started later in the disease stage.

\section{Acknowledgments}

We acknowledge the contribution of Ar Jansen and Diana van Duinen (UMCG) and Wouter Leonhard (LUMC) for their technical support with the animals. Furthermore, we acknowledge Natasha Douglas and Yasmin Farah Cawale for their help and support during the study.

\section{Disclosure Statement}

Prof. Dr. R.T. Gansevoort is a member of the steering committee for the TEMPO and REPRISE clinical trials in which tolvaptan is tested for use in ADPKD [11] and is consultant for Otsuka. Prof. Dr. D.J.M. Peters is also consultant for Otsuka.

\section{References}

1 Neumann HP, Jilg C, Bacher J, Nabulsi Z, Malinoc A, Hummel B, et al: Epidemiology of autosomal-dominant polycystic kidney disease: an in-depth clinical study for southwestern Germany. Nephrol Dial Transplant 2013;28:1472-1487.

2 Harris PC, Torres VE: Genetic mechanisms and signaling pathways in autosomal dominant polycystic kidney disease. J Clin Invest 2014;124:2315-2324.

-3 Yamaguchi T, Pelling JC, Ramaswamy NT, Eppler JW, Wallace DP, Nagao S, et al: cAMP stimulates the in vitro proliferation of renal cyst epithelial cells by activating the extracellular signal-regulated kinase pathway. Kidney Int 2000;57:1460-1471.

4 Mangoo-Karim R, Uchic ME, Grant M, Shumate WA, Calvet JP, Park CH, et al: Renal epithelial fluid secretion and cyst growth: the role of cyclic AMP. FASEB J 1989;3:26292632.

5 Gattone VH 2nd, Maser RL, Tian C, Rosenberg JM, Branden MG: Developmental expression of urine concentration-associated genes and their altered expression in murine infantile-type polycystic kidney disease. Dev Genet 1999;24:309-318.

6 Gattone VH 2nd, Wang X, Harris PC, Torres VE: Inhibition of renal cystic disease development and progression by a vasopressin V2 receptor antagonist. Nat Med 2003;9:1323-1326.
7 Torres VE, Wang X, Qian Q, Somlo S, Harris PC, Gattone VH 2nd: Effective treatment of an orthologous model of autosomal dominant polycystic kidney disease. Nat Med 2004; 10:363-364.

8 Meijer E, Gansevoort RT, de Jong PE, van der Wal AM, Leonhard WN, de Krey SR, et al: Therapeutic potential of vasopressin V2 receptor antagonist in a mouse model for autosomal dominant polycystic kidney disease: optimal timing and dosing of the drug. Nephrol Dial Transplant 2011;26:2445-2453.

-9 Wang X, Gattone V 2nd, Harris PC, Torres VE: Effectiveness of vasopressin V2 receptor antagonists OPC-31260 and OPC-41061 on polycystic kidney disease development in the PCK rat. J Am Soc Nephrol 2005;16:846-851.

10 Aihara M, Fujiki H, Mizuguchi H, Hattori K, Ohmoto K, Ishikawa M, et al: Tolvaptan delays the onset of end-stage renal disease in a polycystic kidney disease model by suppressing increases in kidney volume and renal injury. J Pharmacol Exp Ther 2014;349:258-267.

11 Torres VE, Chapman AB, Devuyst O, Gansevoort RT, Grantham JJ, Higashihara E, et al: Tolvaptan in patients with autosomal dominant polycystic kidney disease. N Engl J Med 2012;367:2407-2418.

12 Knepper MA, Kwon TH, Nielsen S: Molecular physiology of water balance. N Engl J Med 2015;372:1349-1358.
13 Irazabal MV, Torres VE, Hogan MC, Glockner J, King BF, Ofstie TG, et al: Short-term effects of tolvaptan on renal function and volume in patients with autosomal dominant polycystic kidney disease. Kidney Int 2011;80: 295-301.

14 Boertien WE, Meijer E, de Jong PE, Bakker SJ, Czerwiec FS, Struck J, et al: Short-term renal hemodynamic effects of tolvaptan in subjects with autosomal dominant polycystic kidney disease at various stages of chronic kidney disease. Kidney Int 2013;84:1278-1286.

-15 Lantinga-van Leeuwen IS, Leonhard WN, van der Wal A, Breuning MH, de Heer E, Peters DJ: Kidney-specific inactivation of the Pkd1 gene induces rapid cyst formation in developing kidneys and a slow onset of disease in adult mice. Hum Mol Genet 2007;16:3188-3196.

16 Rose BD, Post TW: Hyperosmolal states, hypernatremia; in Clinical Physiology of AcidBase and Electrolyte Disorders, ed 5. United States, The McGraw-Hill Companies, 2001, p 770.

17 Fazekas AS, Funk GC, Klobassa DS, Ruther H, Ziegler I, Zander R, et al: Evaluation of 36 formulas for calculating plasma osmolality. Intensive Care Med 2013;39:302-308.

18 Junqueira LC, Bignolas G, Brentani RR: Picrosirius staining plus polarization microscopy, a specific method for collagen detection in tissue sections. Histochem J 1979;11:447-455. 
19 Zittema D, Boertien WE, van Beek AP, Dullaart RP, Franssen CF, de Jong PE, et al: Vasopressin, copeptin, and renal concentrating capacity in patients with autosomal dominant polycystic kidney disease without renal impairment. Clin J Am Soc Nephrol 2012;7:906-913.

-20 Meijer E, Bakker SJ, van der Jagt EJ, Navis G, de Jong PE, Struck J, et al: Copeptin, a surrogate marker of vasopressin, is associated with disease severity in autosomal dominant polycystic kidney disease. Clin J Am Soc Nephrol 2011;6:361-368.

-21 Boertien WE, Meijer E, Zittema D, van Dijk MA, Rabelink TJ, Breuning MH, et al: Copeptin, a surrogate marker for vasopressin, is associated with kidney function decline in subjects with autosomal dominant polycystic kidney disease. Nephrol Dial Transplant 2012;27:4131-4137.

-22 Boertien WE, Meijer E, de Jong PE, ter Horst GJ, Renken RJ, van der Jagt EJ, et al: Short- term effects of tolvaptan in individuals with autosomal dominant polycystic kidney disease at various levels of kidney function. Am J Kidney Dis 2015;65:833-841.

23 Izumi Y, Nakayama Y, Mori T, Miyazaki H, Inoue $\mathrm{H}$, Kohda Y, et al: Downregulation of vasopressin $\mathrm{V} 2$ receptor promoter activity via V1a receptor pathway. Am J Physiol Renal Physiol 2007;292:F1418-F1426.

24 Machida K, Wakamatsu S, Izumi Y, Yosifovska T, Matsuzaki T, Nakayama Y, et al: Downregulation of the V2 vasopressin receptor in dehydration: mechanisms and role of renal prostaglandin synthesis. Am J Physiol Renal Physiol 2007;292:F1274-F1282.

25 Oi A, Morishita K, Awogi T, Ozaki A, Umezato M, Fujita S, et al: Nonclinical safety profile of tolvaptan. Cardiovasc Drugs Ther 2011; 25(suppl 1):S91-S99.

26 Torres VE, Higashihara E, Devuyst O, Chapman $\mathrm{AB}$, Gansevoort RT, Grantham JJ, et al:
Effect of tolvaptan in autosomal dominant polycystic kidney disease by CKD stage: results from the TEMPO 3:4 trial. Clin J Am Soc Nephrol 2016;11:803-811.

27 Hanoune J, Defer N: Regulation and role of adenylyl cyclase isoforms. Annu Rev Pharmacol Toxicol 2001;41:145-174.

28 Hopp K, Hommerding CJ, Wang X, Ye H, Harris PC, Torres VE: Tolvaptan plus pasireotide shows enhanced efficacy in a PKD1 model. J Am Soc Nephrol 2015;26:39-47.

29 Warner G, Hein KZ, Nin V, Edwards M, Chini CC, Hopp K, et al: Food restriction ameliorates the development of polycystic kidney disease. J Am Soc Nephrol 2016;27:1437-1447.

30 Kipp KR, Rezaei M, Lin L, Dewey EC, Weimbs T: A mild reduction of food intake slows disease progression in an orthologous mouse model of polycystic kidney disease. Am J Physiol Renal Physiol 2016, Epub ahead of print. 